Note

\section{Phage Taillike Particles Found in the Pocks of Streptomyces coerulescens}

\author{
Hikaru Suenaga, Seiya Ogata* \\ and Shinsaku HAYASHIDA

\section{Laboratory of Applied Microbiology, Department of Agricultural Chemistry, Kyushu University, 46, Higashi-ku, Fukuoka 812, Japan}

Received May 2, 1983

We have reported the pock formation of thiostreptonproducing Streptomyces azureus and endomycinproducing Streptomyces endus. ${ }^{1,2)}$ These organisms formed unique colonies or a lawn with pocks on agar media. The pocks appeared spontaneously during the growth of the organisms, and increased in number during subculturings. In the pocks, lysis of the aerial and sporulating hyphae occurred with the production of a large number of phage taillike particles. However, the lysis and the production of these particles did not occur in the substrate hyphae or in any liquid cultures. The occurrence of pocks was detrimental to the cultivation and stock cultures of these organisms or the production of antibiotics. ${ }^{1,3)}$ The pock formation of these organisms has phenotypical features in common with "pocks" of Streptomyces coelicolor $^{4,5)}$ and Streptomyces lividans, ${ }^{6)}$ especially the appearance on the plate of a circular zone (pocks) of sporulation inhibition. However, there are some distinct differences between them, as described elsewhere. ${ }^{1 \sim 3)}$ In the course of screening for other pock-forming Streptomyces, we found that cerulomycin-producing Streptomyces coerulescens formed spontaneously developing pocks similar to those of the above species of
Streptomyces. This paper concerns the pock formation with the production of phage taillike particles in Streptomyces coerulescens.

Streptomyces coerulescens (KCC S-0360) was grown on Rye flakes agar (rye flakes, $1 \%$; glucose, $0.2 \%$; yeast extract, $0.1 \%$; $\mathrm{CaCO}_{3}, 0.2 \%$; agar, $1.5 \%$; $\mathrm{pH} 7.2$ ), for $5 \sim 7$ days at $28^{\circ} \mathrm{C}$. This organism formed a unique confluent lawn with pocks which appeared spontaneously, as shown in Fig. 1. The morphology of the pocks of Streptomyces coerulescens was more similar to that of Streptomyces endus than that of Streptomyces azureus. Spore formation and aerial hyphae growth was inhibited in the pocks. Such inhibition was also seen in the pocks of $S$. azureus and $S$. endus. ${ }^{1 \sim 3)}$

A specimen for electron microscopy was picked up directly from a single pock or overlapping pocks with a platinum loop, then directly suspended in potassium phosphotungstate $(\mathrm{pH} 6.0)$ and placed on grids coated with collodion-carbon, as described in the previous reports. ${ }^{1,2)}$ Electron microscopy showed that almost all hyphae in the pocks were broken or lysed. The same observation was made with $S$. azureus and $S$. endus. ${ }^{1 \sim 3)}$ In the broken. hyphae, or around them, there were numerous phage

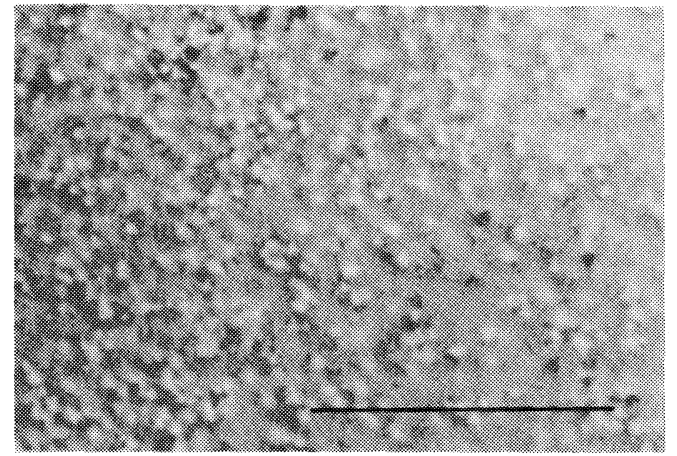

FIG. 1. Spontaneous Appearance of Pocks in Confluent Lawns of Streptomyces coerulescens.

Bar, $1 \mathrm{~cm}$.
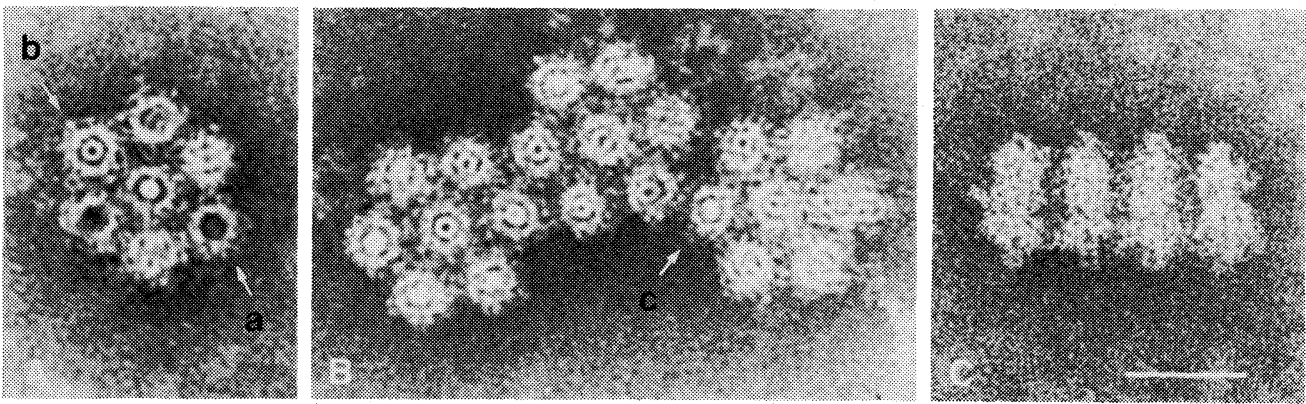

FIG. 2. Electron Micrographs of Hexagonal Crystals and Bullet Shape of Phage Taillike Particles.

Arrow $\mathrm{a}, \mathrm{b}$ and $\mathrm{c}$ show particles with no discernible internal structure, a hollow inner core and a full inner core, respectively. Bar, $50 \mathrm{~nm}$.

* Reprint requests should be addressed to this author. 


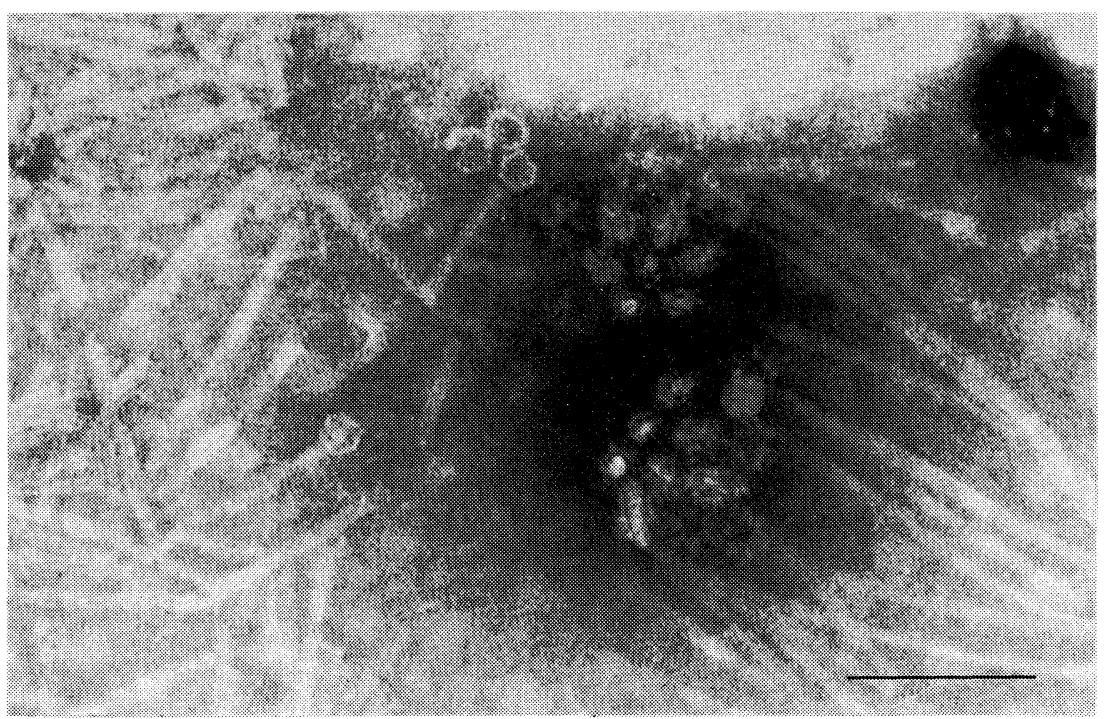

FIG. 3. Electron Micrographs of Long Phage Taillike Particles.

Bar, $100 \mathrm{~nm}$.

taillike particles (short taillike particles), as shown in Fig. 2. Figure $2 \mathrm{C}$ shows the side views of the particles and Fig. $2 \mathrm{~A} \& \mathrm{~B}$ show their end-on views. Their end-on views formed a hexagonal crystal mass, as shown in Figs. 2A and B; a single hexagonal crystal consisted of seven particles. The characteristics of the crystal formation were the same as those of $S$. azureus and $S$. endus. ${ }^{1 \sim 3)}$ Three basic types of the crystals appeared: One (18-nm diameter) had no discernible internal structure (arrow a), the second had a hollow inner core (arrow b), and the third had a full inner core (arrow c: 8-nm in width). In the case of Streptomyces coerulescens, there were other types of particles, long taillike particles of various lengths and 8-nm width, in addition to the short taillike particles (Fig. 2), as shown in Fig. 3. But the production of the long taillike particles was a very low: For instance, 20 to 30 short taillike particles were seen in all electron microscopical observation, whereas the production ratio of long taillike particles to short taillike particles was less than $1 / 1000$. The width of long taillike particles agreed with that of the inner core of short taillike particles. However, the relationship between short taillike particles and long taillike particles is not clear yet. A further study might clarify the relationship between the two types of particles. A small amount of long taillike particles was also found in Streptomyces azureus but not in Streptomyces endus. ${ }^{1,2)}$

Acknowledgments. We are deeply grateful to Dr. A. Seino (KCC Culture Collection of Actinomycetes, Kaken Chemical Ltd.) for his helpful advice and for supplying Streptomyces coerulescens.

\section{REFERENCES}

1) S. Ogata, S. Yoshino, H. Suenaga, K. Aoyama, N. Kitajima and S. Hayashida, Appl. Environ. Microbiol., 42, 135 (1981).

2) S. Ogata, H. Suenaga and S. Hayashida, Appl. Environ. Microbiol., 43, 1182 (1982).

3) S. Ogata, The Actinomycetologist, No. 40, 5 (1982).

4) D. A. Hopwood, K. F. Chater, J. E. Dowing and A. Vivian, Bacteriol. Rev., 37, 371 (1973).

5) M. J. Bibb, J. W. Ward and D. A. Hopwood, Nature (London), 274, 398 (1978).

6) C. J. Thompson, J. M. Ward and D. A. Hopwood, Nature (London), 268, 525 (1980). 